\title{
Physiotherapie korrekt verordnen
}

\author{
Anlässlich zahlreicher Rückmeldungen aus Ärztekreisen vor und insbesondere \\ nach Erscheinen des Artikels «Der Druck steigt - auf Physiotherapeuten und \\ Ärzte» [1] sind Unsicherheiten zur Verordnung von Physiotherapie zutage \\ getreten. physioswiss hat zu dieser Thematik ein Merkblatt publiziert, das \\ Klarheit schaffen soll.
}

\section{Annick Kundert}

Vizepräsidentin Gesundheit, physioswiss
1 Gross B. Der Druck steigt auf Physiotherapeuten und Ärzte. Schweiz Ärztezeitung. 2008;89(23):1016
Korrespondenz: Annick Kundert physioswiss Centralstrasse $8 \mathrm{~b}$ CH-6210 Sursee Tel. 0419266969 Fax 0419266999 info@physioswiss.ch
Auf der Website von physioswiss ist das Merkblatt «Physiotherapie korrekt verordnen» zu finden (www.physioswiss.ch), das die wichtigsten Fragen zur Verordnung von Physiotherapie beantworten soll. Bestehende Unsicherheiten zur Thematik sollen dadurch beseitigt und der administrative Aufwand vermindert werden. Nachfolgend werden die wichtigsten Punkte dieses Merkblatts aufgeführt.

Physiotherapeut(inn)en berechnen ihre Leistungen nach dem Physiotherapietarif.

Beim Physiotherapietarif handelt es sich um einen nationalen und vom Bundesrat genehmigten Vertrag zwischen physioswiss und Santésuisse (gemäss KVG 46 und KLV 5). Als Einzelleistungstarif besteht er aus Sitzungspauschalen und weiteren Zuschlagspositionen.

Grundsätzlich dürfen Ärzte aller Fachrichtungen Physiotherapie verordnen, auch Zahnärzte. Eine Verordnung ist ab Stempeldatum drei Monate gültig. Der Arzt darf pro Verordnung maximal neun Sitzungen ausstellen. Es ist möglich, zwei Behandlungen pro Tag zu verordnen, dies muss vom Arzt aber ausdrücklich auf der Verordnung aufgeführt sein.

Damit Physiotherapeut(inn)en ihre Leistungen von den Versicherern vergütet erhalten, muss auf der Verordnung eine ärztliche (Kurz-) Diagnose bzw. ein Diagnosecode aufgeführt sein, wobei eine globale, unspezifische Diagnose in den meisten Fällen genügt. Bei spezifizierten Diagnosen kann der Arzt die Verordnung ausschliesslich dem Vertrauensarzt zustellen.
Grundsätzlich ist der verordnende Arzt nicht verpflichtet, den Versicherern eine Begründung für die von Physiotherapeut(inn)en gewählten Abrechnungspositionen zu liefern. Diese erfolgt bei aufwendigen Behandlungen allenfalls durch die Physiotherapeut(inn)en.

Die Versicherer können eine schriftliche Präzisierung vom Arzt verlangen, wenn die Kurzdiagnose bzw. der Diagnosecode auf der Verordnung fehlt und in einzelnen Fällen auch dann, wenn eine Präzisierung notwendig ist (z.B. bei Komplexität des Falles oder Lymphödem). Nach Abschluss der Rehabilitationsphasen können Langzeittherapien verordnet werden. Erst dann, also nach Ablauf von vier Behandlungsserien (36 Behandlungen), ist zur gleichen Diagnose eine schriftliche Begründung durch den Arzt notwendig (KLV 5, Abs. 4).

Die Krankenversicherer dürfen die Anzahl Sitzungen pro Patient und Jahr nicht im Voraus festlegen. Diesbezüglich gibt es keine Limitierung durch das KVG pro Patient und Kalenderjahr.

Das Merkblatt «Physiotherapie korrekt verordnen» steht allen Interessierten unter www.physioswiss.ch $\rightarrow$ Services $\rightarrow$ Download \& Links zur Verfügung. Bei weiteren Fragen gibt die Geschäftsstelle von physioswiss gerne Auskunft unter Tel. 041 9266969. 